\title{
ARTICLE
}

\section{Implementation of Photonuclear Reactions in the Monte Carlo Transport Code TRIPOLI-4 and Its First Validation in Waste Package Field}

\author{
Odile PETIT ${ }^{*}$, Nicolas HUOT and Cédric JOUANNE \\ Commissariat à l'Energie Atomique, CEA/DEN/DANS/DM2S/SERMA, 91191 Gif-sur-Yvette Cedex, France
}

\begin{abstract}
This paper presents the implementation of photonuclear reactions in the Monte Carlo transport code TRIPOLI-4. A complete coupling of neutron and photon transport is thus achieved by this feature which can be used with the electromagnetic shower option as well. The production of photoneutrons can occur when photon energy is above a threshold of a few MeV. The recent photonuclear data from the ENDF/B-VII library were chosen for this work. The conventions assumed for the reading of these data are first listed. The general algorithm of photon collision and its non analog part for photonuclear reactions sampling are then detailed. Analytical expressions used for the sampling of photoneutrons from an individual photonuclear reaction are also given. At last, a simulation of a waste package, involving a complete coupling of electron, positron, photon, and neutron transport, was carried out. The test case consists in an 11-MeV electron beam produced by an accelerator, generating high energy photons by Bremsstrahlung on a tungsten target, and then photoneutrons by photofission on an active filter embedded in the waste package. The results of the TRIPOLI-4 simulation are compared to MCNPX results and to measurement values. A good agreement is shown.
\end{abstract}

KEYWORDS: photonuclear reactions, Monte Carlo simulation, radioactive wastes measurement

\section{Introduction}

TRIPOLI-4® ${ }^{\mathrm{a}}$ Monte Carlo neutron, photon, electron, and positron transport code has been developed and validated at Commissariat à l'Energie Atomique $(\mathrm{CEA}){ }^{1)}$ Its main application fields cover radiation shielding, criticality safety, nuclear instrumentation and nuclear reactor physics. Coupled neutron-photon transport was already implemented in this code, but up to now the production of photoneutrons through photonuclear reactions was not taken into account.

These reactions can occur above a threshold of a few $\mathrm{MeV}$ : generally between 5 and $7 \mathrm{MeV}$, it can be lower (from 2 to $5 \mathrm{MeV}$ ) for certain light nuclides as for the deuterium $(2.2 \mathrm{MeV})$ or the beryllium 9 isotope $(1.7 \mathrm{MeV})$. The effect of the photonuclear reactions in several fields (nuclear instrumentation, dosimetry, but also reactor shielding calculations in Beryllium and heavy water environments) has already been noticed in the literature. ${ }^{2,3)}$

Section II is devoted to the choice of a photonuclear data library and to different conventions assumed for the reading of these data by the code. The details of the implementation of the photoneutrons sampling are then given in Section III. The last section presents an example of validation on a waste package set-up, showing comparisons of TRIPOLI-4 results with MCNPX results and experimental values.

\section{Photonuclear Data Management}

\section{Choice of a Photonuclear Data Library}

Several libraries of photonuclear data are currently avail-

*Corresponding author, E-mail: odile.petit@cea.fr

${ }^{a}$ TRIPOLI-4® is a registered trademark of CEA.

(C) 2011 Atomic Energy Society of Japan, All Rights Reserved. able from Russia (BOFOD-99 library), China (CNDC library), Japan (JENDL library), South Korea (KAERI library) and USA (ENDF/B-VII library). The IAEA Photonuclear Data Library ${ }^{4)}$ gives also access to an international collection of several libraries. It should be noted that all these photonuclear data libraries are far less complete than the standard neutronic and photoatomic ones widely used by Monte Carlo codes; photonuclear data are delivered for far less nuclides and for most of them only a small number of reactions are given. All libraries are globally using the ENDF/B format ${ }^{5)}$ but different conventions can be found (e.g., on the MT numbers related to certain photonuclear reactions). We have chosen to work with the ENDF/B-VII library ${ }^{6)}$ produced by the Los Alamos National Laboratory (LANL, USA), delivering photonuclear data for a relatively large number of nuclides (163) and being relatively recent.

\section{Conventions Assumed for the Data Reading}

For a given nuclide, TRIPOLI-4 needs all continuous energy cross-sections and energy or energy-angle distributions available for the different photonuclear reactions present in the evaluated data file.

The total photonuclear cross-section is first given either under MT number 3 or 5 (if the previous one is absent). This cross-section is read and automatically added by the code to the total photoatomic one (which is read in an independent EPDL $^{7)}$ evaluated data file) to get the total photon cross-section. A common energy grid is build for this summation.

Different individual photonuclear reactions can then be found separately. We list here those which are taken into account by TRIPOLI-4 if their associated data are present in 
the file:

- $\quad(\gamma, n)$ reactions, including several discrete inelastic levels with MT numbers ranging from 50 (for the ground state) to 90 (generally only a few levels are present), and the continuous inelastic given by MT number 91 ;

- $\quad(\gamma, 2 n),(\gamma, 3 n),(\gamma, n \alpha)$ and $(\gamma, n p)$ reactions, given by the following MT numbers: 16, 17, 22 and 28;

- $\quad(\gamma, \mathrm{f})$ photofission for fissile nuclides, given by MT number 18;

- photoabsorptions which do not emit any neutron, given by the MT numbers 102 to 107 and 111;

- a complement to the total photonuclear, given under MT number 5 (unlike the case of neutronic evaluated data files, it may gather a lot of reactions listed above but not present individually in the file, or even be a total photonuclear).

Photofission multiplicities are also read if needed, under the following MT numbers: 452 for the total yield, 455 for the delayed yield and 456 for the prompt yield.

Additionally, for each of the reactions present in the evaluated data file, data related to the emitted neutrons are read either through an energy distribution or a double differential energy-angle distribution. However, neutron emission data are not always present for all reactions whose cross-sections have been tabulated. In such a case, the isotropy hypothesis can be done only for the discrete inelastic $(\gamma, n)$ levels. Otherwise, the reaction is ignored by the code.

\section{Implementation of the Photoneutrons Sampling in TRIPOLI-4 Code}

\section{Non Analog Sampling of Photonuclear Reactions}

Photonuclear events are rare when compared to photoatomic ones, such as photoelectric effect, Compton scattering or pair production. Photonuclear cross-sections are actually several orders of magnitude lower than photoatomic ones for all nuclides; their maxima do not exceed a few hundreds of millibarns. Therefore, an analog sampling of photonuclear reactions in the simulation of photon collisions would lead to a huge computation time necessary to achieve satisfactory statistics on photoneutrons production.

To overcome this problem, a non analog sampling was implemented; at each photon collision, the sampling of a photonuclear reaction is forced and hence a correction on the statistical weights of the emitted photoneutrons is done accordingly.

Photoabsorption reactions which do not emit any neutron are not sampled either (the idea is similar to the implicit capture used for neutron collisions sampling for example), and thus another weight correction is applied in compensation.

\section{Collision Operators}

(1) Analog Operator for the Global Photon Collision

At each photon collision, the analog operator can be written as follows:

$$
\begin{aligned}
C\left(r, E^{\prime} \rightarrow E, \Omega^{\prime} \rightarrow \Omega\right) & =\sum_{i} N_{i}(r) \times\left[\sum_{j} \sigma_{P N_{j i}}\left(E^{\prime} \rightarrow E, \Omega^{\prime} \rightarrow \Omega\right)\right. \\
& \left.+\sum_{k} \sigma_{P A_{k i}}\left(E^{\prime} \rightarrow E, \Omega^{\prime} \rightarrow \Omega\right)\right] \times \frac{1}{\Sigma_{t}\left(r, E^{\prime}\right)},
\end{aligned}
$$

where the microscopic cross-section indices $P N_{j i}$ and $P A_{k i}$ stand for the photonuclear reaction of type $j$ and the photoatomic reaction of type $k$ for nuclide $i, \Sigma_{t}\left(r, E^{\prime}\right)$ is the total photon macroscopic cross-section at point $r$ and energy $E$, and $N_{i}(r)$ is the atom density of nuclide $i$ at point $r$.

(2) Non Analog Photonuclear Collision Operator

The photonuclear part of Eq. (1) for the chosen nuclide $i$ would be given by

$$
C_{P N_{i}}\left(E^{\prime} \rightarrow E, \Omega^{\prime} \rightarrow \Omega\right)=\sum_{j} \frac{\sigma_{P N_{j i}}\left(E^{\prime} \rightarrow E, \Omega^{\prime} \rightarrow \Omega\right)}{\sigma_{\gamma_{i i}}\left(E^{\prime}\right)},
$$

where $\sigma_{\gamma_{t i}}\left(E^{\prime}\right)$ is the total photon cross-section for nuclide $i$ at energy $E$ '. Thus, when sampling the photonuclear reaction $j$, the statistical weight of the emitted neutron would be

$$
W_{n}=v_{j i}\left(E^{\prime}\right) \times W_{\gamma}
$$

taking into account the statistical weight $W_{\gamma}$ of the incident photon and the neutron multiplicity $v_{j i}\left(E^{\prime}\right)$ of the chosen photonuclear reaction $j$ for nuclide $i$ at the energy $E$ ' of this photon.

In order to improve the variance of photonuclear events, as said in Section III. 1, we introduce Eqs. (4) to (5). Instead of Eq. (2), we use the following non analog operator:

$$
\widetilde{C}_{P N_{i}}\left(E^{\prime} \rightarrow E, \Omega^{\prime} \rightarrow \Omega\right)=\sum_{j \neq a b s_{P N}} \frac{\sigma_{P N_{j i}}\left(E^{\prime} \rightarrow E, \Omega^{\prime} \rightarrow \Omega\right)}{\sigma_{(\gamma, n x) i}\left(E^{\prime}\right)}
$$

where the cross-section index $a b s_{P N}$ stands for photonuclear reactions which do not emit any neutron and $\sigma_{(\gamma, n x) i}\left(E^{\prime}\right)$ is the sum of all photonuclear reactions different from this kind for nuclide $i$ at energy $E$ '. Consequently, the statistical weight of the emitted neutron becomes

$$
\widetilde{W}_{n}=\frac{\sigma_{(\gamma, n x) i}\left(E^{\prime}\right)}{\sigma_{\gamma_{i i}}\left(E^{\prime}\right)} v_{j i}\left(E^{\prime}\right) \times W_{\gamma},
$$

which can be rather split in several neutrons, using the multiplicity factor. Sampling a photonuclear reaction from Eq. (4) at each photon collision and applying the weight correction given by Eq. (5) allows to achieve the non analog sampling introduced in Section III. 1.

With a similar idea, photofission sampling can also be biased, being forced at each photon collision instead of being sampled among all possible photonuclear reactions according to the operator $\widetilde{C}_{P N_{i}}$. Further corrections are then needed on Eqs. (4) and (5).

\section{Photoneutrons Emission Energy and Direction Sam- pling}

(1) Discrete Inelastic $(\gamma, n)$ Levels

For the sampling of photoneutrons from a chosen level of discrete inelastic $(\gamma, \mathrm{n})$ reaction, the following expression of the neutron energy in the center of mass system is first used: $:^{8)}$ 


$$
\hat{E}_{n}=\frac{A-1}{A}\left(E_{\gamma}-Q^{\prime}-\frac{E_{\gamma}{ }^{2}}{2 m_{n} A}\right),
$$

where $A$ is the mass number ratio (i.e. nucleus mass in neutron mass unit) of the collided nuclide, $E_{\gamma}$ is the energy of the incident photon, $Q^{\prime}$ is the opposite of the reaction $\mathrm{Q}$ value given in the evaluated data and $m_{n}$ is the neutron mass, given in energy equivalent unit $\left(m_{n}=939.565 \mathrm{MeV}\right)$.

Let $\mu$ be the cosine between the incident photon direction and the emitted neutron direction, and $\hat{\mu}$ the same cosine given in the center of mass system. The value of the latter is sampled from the equiprobable cosine tables processed by TRIPOLI-4 from the angular distribution of the chosen level.

Then, the neutron energy is calculated in the laboratory system through

$$
E_{n}=\frac{A-1}{A}\left(E_{\gamma}-Q^{\prime}\right)-\frac{(A-2) E_{\gamma}^{2}}{2 m_{n} A^{2}}+\frac{1}{A} \sqrt{\frac{2(A-1)}{m_{n} A}\left(E_{\gamma}-Q^{\prime}-\frac{E_{\gamma}{ }^{2}}{2 m_{n} A}\right)} E_{\gamma} \hat{\mu}_{.} .
$$

Hence, from $\hat{\mu}$ value and Eqs. (6) and (7), $\mu$ is also obtained in the laboratory system:

$$
\mu=\sqrt{\frac{\hat{E}_{n}}{E_{n}}} \hat{\mu}+\frac{E_{\gamma}}{A \sqrt{2 m_{n} E_{n}}} .
$$

(2) Double Differential Distributions

The neutron energy and direction sampling through double differential distributions is similar to the sampling already used for double differential neutron scattering reactions. However, when these data are given in the center of mass system, expressions specific to the photonuclear case must be derived:

$$
E_{n}=\hat{E}_{n}+\frac{1}{A} \sqrt{\frac{2 \hat{E}_{n}}{m_{n}}} E_{\gamma} \hat{\mu}+\frac{E_{\gamma}^{2}}{2 m_{n} A^{2}}
$$

and $\mu$ is given by the same expression as in Eq. (8).

Moreover, when Kalbach-Mann systematics distributions are used, ${ }^{5)}$ some of the expressions in the previous reference, detailed only for the neutron scattering case, must be adapted to the photonuclear case.

\section{First Validation on a Realistic Case}

\section{Methodology}

One of the most important applications of photonuclear physics is the non-destructive control of nuclear waste for their management or non proliferation. The Instrumental Photon Activation Analysis (IPAA) consists in irradiating actinides with high energy photons produced by an electron accelerator associated to a Bremsstrahlung conversion target (tungsten). These photons are highly penetrating and induce photofission reactions in the package. Delayed photoneutrons are counted between accelerator pulses using ${ }^{3} \mathrm{He}$ counters inserted in polyethylene detection units to thermalize photoneutrons. The polyethylene is surrounded by cadmium to cut external low energy neutrons. Detailed descriptions of the method can be found in Reference 9.

The validations use a true waste package which has been measured with both IPAA and destructive analysis as refer-

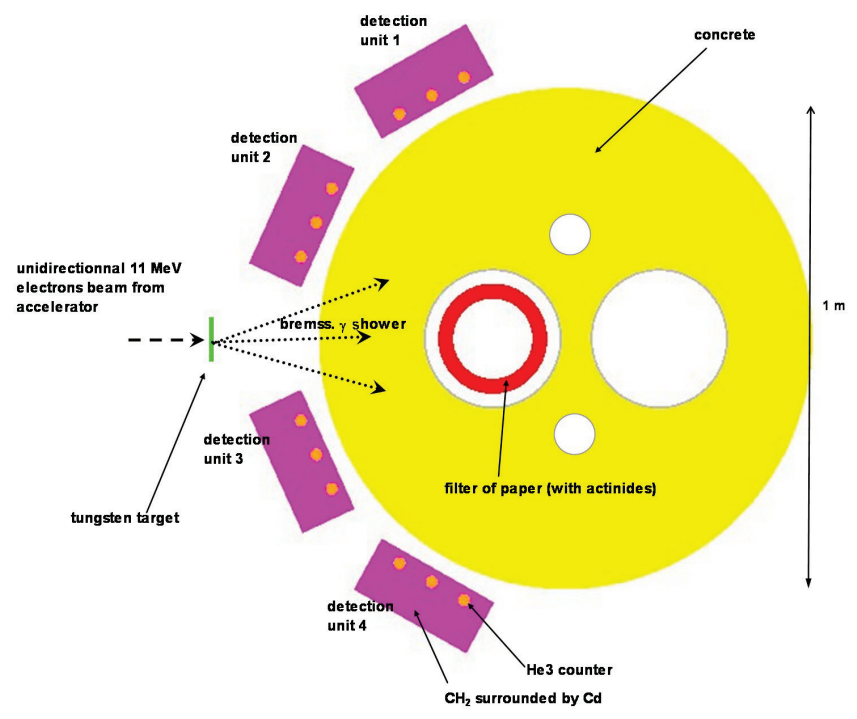

Fig. 1 Layout of experimental geometry simulated

ence. The contaminated case is composed of a cylindrical paper filter (containing $4 \mathrm{~g}$ of uranium) surrounded by a honeycombed case of stainless steel. All is set inside a stainless steel case which is embedded inside the concrete cylindrical package (whose height and diameter are $1 \mathrm{~m}$ ). The three other cases are displayed as well to make simulations as realistic as possible but they contain no actinides. The package is surrounded by four detection units. The high energy photons are produced by $11 \mathrm{MeV}$ electrons coming from a linear accelerator. ${ }^{9)}$ Figure 1 shows the experimental setup.

Two kinds of validation have been carried out.

First a code-code comparison between MCNPX 2.5.0 and TRIPOLI 4.7 has been made with simplified physics.

Secondly, calculations have been performed in full mode $\mathrm{e}^{ \pm}, \gamma, \mathrm{n}$ in order to compare with experiments.

All nuclear and photonuclear data used in both codes come from ENDF/B-VII library. Concerning photonuclear data, only those of ${ }^{238} \mathrm{U}$ nuclide were used for these calculations.

\section{Comparison of MCNPX 2.5.0 and TRIPOLI 4.7 Re- sults with a Simplified Photon Beam}

MCNPX 2.5.0 and TRIPOLI 4.7 results are compared using only a unidirectional photon probe beam with an average Bremsstrahlung spectrum with energy ranging from $5 \mathrm{MeV}$ (about photofission threshold) to $11 \mathrm{MeV}$. This enables code comparison without including Bremsstrahlung model (only photons and neutrons are tracked). However, comparison to experimental results can not be achieved in this way.

Due to parallelism computer limitations and codes differences both codes have not been run with the same running options (for example photon forced collisions have been used in MCNPX whereas more source particles have been simulated with TRIPOLI to achieve a similar convergence), therefore computer time comparison is not meaningful. Calculations have been performed on 64 to 256 Itanium core $1.6 \mathrm{GHz}$ during about $24 \mathrm{~h}$. 
Table 1 Comparison between TRIPOLI 4.7 and MCNPX results with a simplified photon beam

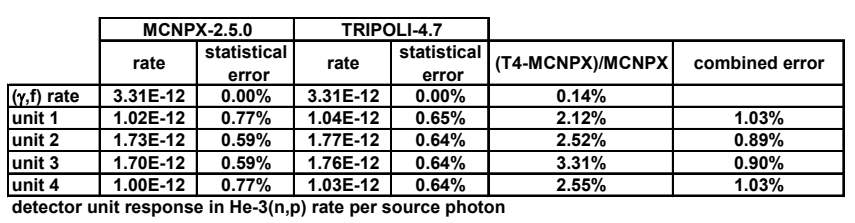

The compared scores are:

- $(\gamma, \mathrm{f})$ rate in the filter, which tests both physical high energy photon transport and $(\gamma, f)$ cross section access.

- ${ }^{3} \mathrm{He}(\mathrm{n}, \mathrm{p})$ rate in the detection units which gives directly measurable information related to total photoneutron production and transport.

Table 1 gives the results.

We have no difference on the $(\gamma, f)$ reaction rate, showing the absence of bias related to photon transport or photofission sampling, since the differences of reaction rate in the detection units do not exceed 3\% and remain within 3 standard deviations.

\section{Comparison of MCNPX 2.5.0 and TRIPOLI 4.7 with the Accelerator Electron Beam Experiment}

Comparisons with experimental counts need to take into account all timing data from accelerator pulse sequences. The photoneutron counting period occurs from $14.4 \mathrm{~ms}$ after the electron pulse to $38.4 \mathrm{~ms}$. The next pulse arrives at $40 \mathrm{~ms}$ (recurrence frequency is $f_{r}=25 \mathrm{~Hz}$ ). The total duration of the measurement is 1 hour. Figure 2 shows the temporal scheme, where $t_{c}$ is the counting duration, $t_{d}$ is the delay between the end of the pulse and the beginning of counting and $t_{i}$ is the macro pulse duration.

In ENDF/B-VII library, the only delayed data of photofission that we used in our calculation was the multiplicity $v_{d e l}$. Moreover, only photonuclear reactions on ${ }^{238} \mathrm{U}$ nuclide were included in the simulation because there is no other delayed photoneutron sources at photon energies below $15 \mathrm{MeV}$. Due to the fact that ${ }^{3} \mathrm{He}$ detectors carry out an integral count of fast photoneutron thermalized in polyethylene detection units, one can, in first approximation, renormalize the total uranium photoneutron count from simulation as shown below assuming that prompt and delayed photoneutron energy spectra are similar with respect to a gross fast neutron counting:

$$
\text { Count }_{3_{H e(n, p)}}=\tau_{3_{H} \text { He(n,p)simulated }} \underbrace{\left(\frac{\tau_{\sigma(\gamma, f)}}{\tau_{\sigma(\gamma, p h n t o t)}}\right)}_{\text {photofission ratio }} \underbrace{\left.\frac{\tau_{\sigma(\gamma, f)}}{\tau_{v_{p r} \sigma_{(\gamma, f)}}+\tau_{v_{\text {del }} \sigma_{(\gamma, f}}}\right)}_{\text {mean multiplicity ratio }} F_{k i n} \text {, }
$$

where $\tau_{\sigma(\gamma, p h n t o t)}$ stands for the total uranium photonuclear reaction rate, $\tau_{\sigma(\gamma, f)}$ stands for the photofission reaction rate, $\tau_{\text {prt } \sigma(\gamma, f)}$ and $\tau_{v \operatorname{del} \sigma(\gamma, f)}$ are the corresponding prompt and delayed photofission neutron production rate. $\tau_{3 H e(n, p) \text { simulated }}$ is the simulated ${ }^{3} \mathrm{He}(\mathrm{n}, \mathrm{p})$ rate normalized to the accelerator electron current. ${ }^{9,10)}$

In Eq. (10), the detector count is proportional to the reaction rate of ${ }^{3} \mathrm{He}$ normalized to one neutron produced by

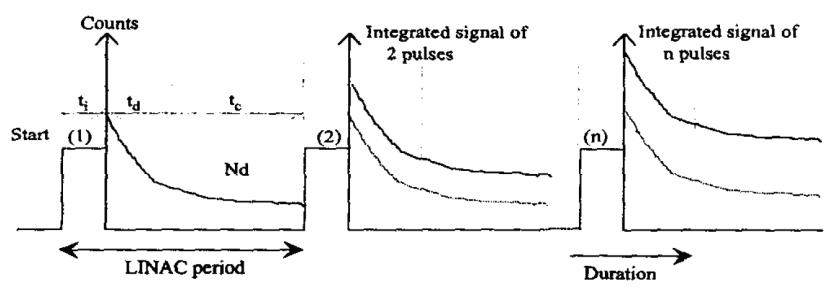

Fig. 2 Layout of delayed photoneutron counting ${ }^{9)}$

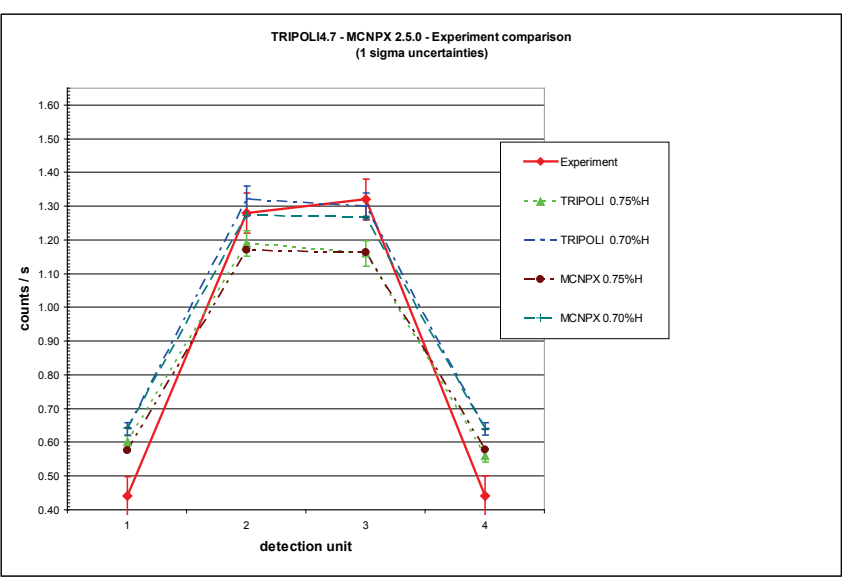

Fig. 3 Comparison of TRIPOLI 4.7 and MCNPX 2.5.0 in full mode calculation $\left(\mathrm{e}^{ \pm}, \gamma, \mathrm{n}\right)$ with experiment

photofission, weighted by the kinetic factor $F_{k i n}$ as detailed below. Normalization to one neutron of photofission is obtained by multiplying the calculation by photofission fraction and dividing by the related multiplicity.

Kinetic simulation as shown in Fig. 2 can not be carried out by MCNPX 2.5.0 and TRIPOLI 4.7, and thus it is taken into account separately by the factor $F_{k i n}$, sum of the number of delayed neutrons emitted by group $i$ during time $t_{c}$ over the 6 groups $\left(\beta_{i}, \lambda_{i}\right)$ during $n$ accelerator pulses. $\beta_{i}$ and $\lambda_{i}$ are the usual kinetic parameters. ${ }^{11)}$

$$
F_{k i n}=\sum_{i=1}^{6} \frac{N_{d}(i)}{\left(1-\omega_{i}\right)^{2}}\left[(n+1)\left(1-\omega_{i}\right)-\left(1-\omega_{i}^{n+1}\right)\right]
$$

where

$$
\omega_{i}=e^{-\lambda_{i} / f_{r}}
$$

and

$$
N_{d}(i)=\frac{\beta_{i}}{\lambda_{i}}\left(1-e^{-\lambda_{i} t_{i}}\right) e^{-\lambda_{i} t_{d}}\left(1-e^{-\lambda_{i} t_{r}}\right)
$$

Figure 3 shows the experimental results in counts per second in each detection unit after subtraction of active and passive noise from the raw signal and corresponding calculation results with MCNPX 2.5.0 and TRIPOLI 4.7.

Photoneutron transport is very sensitive to humidity concentration in the package concrete. Figure 3 shows calculations for two proportions of hydrogen rate $0.75 \%$ and $0.70 \%$ in mass corresponding to humidity rate of $6.6 \%$ and $6.3 \%$ (water content as a percentage of the concrete mass). 
Table 2 Comparison Experiment - Simulation on detection units 2 and 3 (hydrogen rate of $0.70 \%$ )

\begin{tabular}{|c|c|c|c|c|}
\hline & \multicolumn{2}{|c|}{ detection unit 2} & \multicolumn{2}{|c|}{ detection unit 3} \\
\hline & counting $(\mathbf{c} / \mathbf{s})$ & uncertainty $1 \sigma$ & counting (c/s) & uncertainty $1 \sigma$ \\
\hline MCNPX 2.5.0 & 1.27 & $2.3 \%$ & 1.27 & $2.2 \%$ \\
\hline TRIPOLI 4.7 & 1.32 & $3.3 \%$ & 1.30 & $3.3 \%$ \\
\hline Experiment & 1.28 & $4.6 \%$ & 1.32 & $4.6 \%$ \\
\hline
\end{tabular}

$0.75 \%$ is the common hydrogen proportion for such kind of concrete whereas $0.70 \%$ is the average proportion from sample chemical analysis. The two codes give results in agreement within $1 \sigma$ uncertainty and exhibit the high sensitivity to humidity concentration, taking into account concrete thickness of several tens of centimeters, $0.05 \%$ of difference on hydrogen gives about $10 \%$ of neutron counting. The proportion of $0.70 \%$ is close to the sample measurements and gives simulation results that are close to experimental values. Detection unit 1 and 4 are more distant from the filter and therefore their results are more sensitive to humidity gradient. Due to the fact that they are close to detection limit $(0.3 \mathrm{c} / \mathrm{s}),{ }^{10)}$ they are excluded from the actinide quantification.

Table 2 shows final results on detection units 2 and 3. The differences between the Monte Carlo codes (TRIPOLI as well as MCNPX) and the experiment are about 2 to $3 \%$ which is far below Monte Carlo standard deviation and experimental uncertainties. Finally, this shows a good agreement between TRIPOLI, MCNPX and experiment in full transport mode including all particles and almost all physics available in the code.

\section{Conclusion}

In this paper the implementation of photonuclear reactions in the TRIPOLI-4 Monte Carlo code is detailed, as available in the version 7 of the code. As a first validation of this feature, simulation results obtained in a complete coupling of electron, positron, photon and neutron transport are then successfully compared to MCNPX 2.5.0 results and experimental values.

\section{Acknowledgment}

The authors gratefully acknowledge Electricité de France (EDF) and AREVA support.

\section{References}

1) O. Petit, F. X. Hugot, Y. K. Lee, C. Jouanne, TRIPOLI-4 version 4 user guide, CEA-R-6169, Commissariat à l'Energie Atomique (CEA), France (2008).

2) M. White, R. Little, M. Chadwick, Photonuclear physics in MCNP(X), LA-UR-99-4827, Los Alamos National Laboratory (LANL), USA (1999).

3) F. Gallmeier, "Photoneutron production in MCNP4A," Proc. ANS RPSD., No. Falmouth, Massachusetts, USA, Apr. 21-25, 1996, 780-786 (1996).

4) IAEA Nuclear Data Services, http://www-nds.iaea.org/photonuclear

5) Cross Section Evaluation Working Group, ENDF-102 Data formats and procedures for the evaluated nuclear data file ENDF-6, BNL-NCS-44945, National Nuclear Data Center, Brookhaven National Laboratory (1990).

6) M. Chadwick et al., "ENDF/B-VII.0: Next generation evaluated nuclear data library for nuclear science and technology," Nucl. Data Sheets, 107, 2931-3060 (2006).

7) IAEA Nuclear Data Services, http://www-nds.iaea.or.at/epd197

8) A. Wattenberg, "Photo-neutrons sources and energy of the photo-neutrons," Phys. Rev., 71[8], 497-507 (1947).

9) N. Saurel, J. M. Capdevila, N. Huot, M. Gmar, "Experimental and simulated assay of actinides in a real waste package," Nucl. Instr. Meth. Phys. Res., A550, 691-699 (2005).

10) N. Saurel, J. M. Capdevila, N. Huot, "Tomography Applied to Actinides Detection by Photofission," 2000 IEEE Nucl. Sci. Symp., Conference Record, 3, 27/21-27/26 (2000).

11) O. Nikotin, K. Petrzhak, "Delayed neutrons in the photofission of heavy nuclei," Sov. At. Energy, 20, 300 (1966). 